\title{
O021: Infection control enclosure (ICE) POD: meeting the need for more single rooms
}

\author{
J Salkeld \\ From 2nd International Conference on Prevention and Infection Control (ICPIC 2013) \\ Geneva, Switzerland. 25-28 June 2013
}

\begin{abstract}
Introduction
An increase in single occupancy rooms reduces transmission, improves hand hygiene compliance and increases patient satisfaction. Thus, there is a demand for more single rooms, particularly in hospitals with a low proportion of single rooms as is common in Europe. Furthermore, where the previous room occupant was infected or colonised with a pathogen, the subsequent admission is significantly less likely to acquire that pathogen if the room is decontaminated using hydrogen peroxide vapour (HPV) rather than being cleaned and disinfected using standard methods.
\end{abstract}

\section{Objectives}

Bioquell has developed the 'Infection Control Enclosure' (ICE)-pod, a bespoke, semi-permanent structure with a door and an integral air handling system to provide a negative air-flow, which can be erected around existing bed spaces without closing wards.

\section{Methods}

The ICE-pod combines many of the benefits of single rooms whilst retaining the primary advantages of bays /open wards. The pods can also be sealed for HPV decontamination.

\section{Results}

ICE-pods can be used to interrupt the spread of nosocomial pathogens by providing additional capacity to segregate patients known to be infected or colonised with pathogens, for pre-emptive segregation of high-risk patients, to provide a single room environment for patients that require high visibility such as those at risk of falls, and the ability to decontaminate individual bed spaces using HPV. The ICE-pod will also improve the privacy and dignity of patients cared for in multi-occupancy areas, and has the potential to "free up" side rooms for patients requiring additional privacy and dignity. There are tangible potential cost-benefit advantages associated with accelerated discharge from intensive care units and other high-cost units and avoiding the high cost of permanent conversion programs. The ICE-pod will provide an overall improvement in the flexibility of patient flow throughout a hospital, which will increase throughput and decrease the number of patients placed temporarily in sub-optimal specialties.

\section{Conclusion}

Implementation is currently underway in the UK and will include assessment of patient and staff acceptability of the ICE-pod. Further trials to evaluate the clinical impact of the ICE-pod are planned.

\section{Disclosure of interest}

J Salkeld: Employee of Bioquell.

Published: 20 June 2013

doi:10.1186/2047-2994-2-S1-O21

Cite this article as: Salkeld: O021: Infection control enclosure (ICE) POD: meeting the need for more single rooms. Antimicrobial Resistance and Infection Control 2013 2(Suppl 1):O21. 